\title{
Joint Denoising and Distortion Correction for Atomic Column Detection in Scanning Transmission Electron Microscopy Images
}

\author{
Chenyu Zhang ${ }^{1}$, Benjamin Berkels ${ }^{2}$, Benedikt Wirth ${ }^{3}$ and Paul M. Voyles ${ }^{1}$ \\ 1. Department of Materials Science and Engineering, University of Wisconsin-Madison, Madison, \\ Wisconsin, United States \\ 2. AICES Graduate School, RWTH Aachen University, Germany \\ 3. Applied Mathematics, University of Münster, Germany
}

Atomic column positions in images of scanning transmission electron microscopy (STEM) contain important information about material structure and properties, but the precision with which those columns can be located is often limited by noise in the measured intensities and by scan distortion. Several previous works (e.g. [1-3]) have used post-processing methods on a series of STEM images to obtain single high signal-to-noise ratio, reduced distortion images. Then, from those images, precise column positions can be obtained. These methods are effective, yielding precision in the position of atomic columns down below $1 \mathrm{pm}$ [1], but at very high dose to the sample. Here, we propose an alternative approach that combines denoising and distortion correction, then directly identifies columns positions from noisy image series. A high-quality image is never generated to predict atomic column positions. We call this method joint denoising and distortion correction (JDDC).

JDDC assumes that the perfect, noiseless and undistorted high-resolution STEM image can be represented as a sum of two-dimensional Gaussian peaks. Then, it seeks to find the set of parameters describing those peaks, the sample drift from image to image, and a set of distortions within each image that reproduce the intensity pattern within each experimental image. The parameters are discovered using variational methods to maximize the Bayesian probability of the column distribution, drift pattern, and distortions, given the noisy experimental data. Noise is modeled as a mixture of Poisson and Gaussian noise, Brownian motion is used to model the sample drift, and distortions include flyback error and probe jitter. The mathematical model is described in detail elsewhere [4].

JDDC has been tested on series of 50 high-angle annular dark-field (HAADF) images acquired from a $\mathrm{SrTiO}_{3}$ sample using a $\mathrm{C}_{\mathrm{s}}$-corrected FEI Titan microscope. Fig. 1(a) shows the JDDC-derived positions of $\mathrm{Sr}$ (red) and $\mathrm{Ti}$ (blue) columns superimposed on the first raw frame of the data series. Fig. 1(b) shows the ideal image calculated from the JDDC positions, and Fig 1(c) shows that ideal image corrupted by the JDDC drift and distortion pattern. A zoomed in view of Figs. 1(a) and 1(c) shows good agreement on the distorted peak shapes, which suggests that the JDDC method is capturing the correct drift and distortion.

As a benchmark test, the same $\mathrm{SrTiO}_{3} \mathrm{HAADF}$ image series with 16 frames is processed using our stateof-the-art non-rigid registration (NRR) scheme to obtain a high-quality image [1], and the atomic column positions were determined from that image by fitting to two-dimensional Gaussians. The performance of the two methods is judged based on precision defined as the standard deviation of many measurements of the same interatomic spacing in the image [5]. Fig. 2(a) compares the precision of the two methods under different total electron dose by changing the dwell time during image acquisition. JDDC produces precision better than $3.5 \mathrm{pm}$ for a wide range of electron dose, and it outperforms NRR when the electron dose is less than $20000 \mathrm{e}^{-} / \AA^{2}$. This dose is still high by biological standards, but it yields quite noisy single images by materials science standards, as shown in Fig. 2(b). Thus, JDDC is a step towards achieving higher precision and lower dose in STEM imaging [6]. 
References:

[1] A. B. Yankovich et al, Nat. Commun. 5 (2014), p. 4155.

[2] X. Sang and J. M. LeBeau, Ultramicroscopy 138 (2014), p. 28.

[3] L. Jones et al, Adv. Struct. Chem. Imaging 1 (2015), p. 8.

[4] B. Berkels and B. Wirth, https://arxiv.org/abs/1612.08170 (2016).

[5] S. Bals et al, Phys. Rev. Lett. 96 (2006), p. 096106.

[6] Research at UW-Madison (CZ and PMV) was supported by the US Department of Energy (DE-

FG02-08ER46547). Research at RWTH (BB) was supported by the Excellence Initiative of the German Federal and State Government. Research at Münster (BW) was supported by the Alfried Krupp Prize for Young University Teachers awarded by the Alfried Krupp von Bohlen und Halbach-Stiftung.
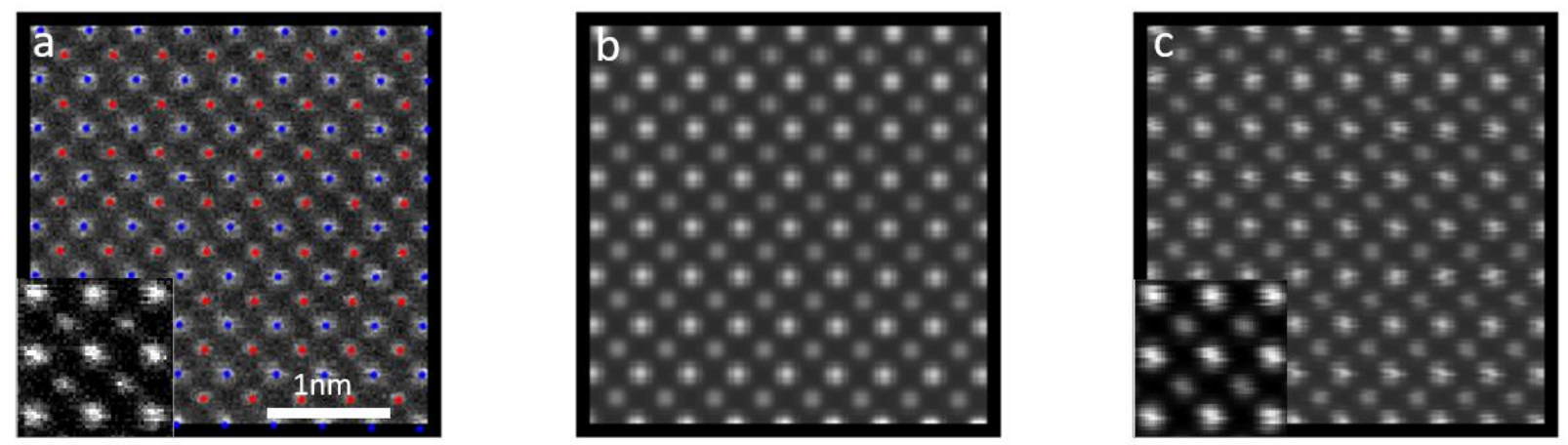

Figure 1. (a) Atomic columns detected by the JDDC. Red marks Sr columns and blue marks Ti. (b) The ideal, distortion-free image reconstructed from the JDDC-derived atomic column parameters (c) The ideal image in (b) with the JDDC-derived drift and distortion applied.
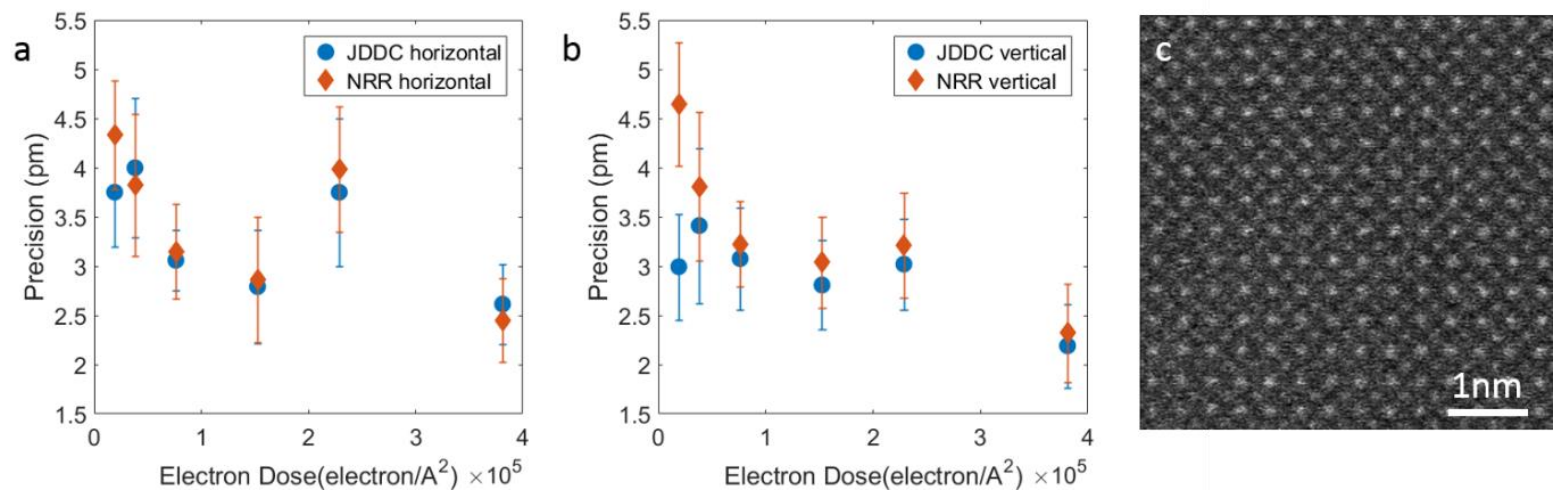

Figure 2. (a)(b) Performance of JDDC and NRR under different total electron dose for horizontal and vertical direction. Each data point is averaged from at least 11 tests, each with 16 input frames. (c) Example of one single frame from the noisiest data set 